\title{
Multi-tasking and inequity aversion in the linear-exponential-normal moral hazard model
}

\author{
Bartling, Björn
}

\begin{abstract}
This paper analyzes the impact of wage comparisons among inequity-averse agents on optimal incentive intensities in a linear-exponential-normal moral hazard model with multi-tasking. We consider individual and team production tasks that differ in that only individual production causes wage inequality. If the tasks are substitutes in the agents' effort cost functions, the principal might want to balance incentives and reduce the agents' overall inequality exposure. We show that team production incentives can then be muted below the level that results from noisy measurement and risk aversion alone- even though team production does not cause wage inequality.
\end{abstract}

DOI: https://doi.org/10.1016/j.econlet.2012.04.010

Posted at the Zurich Open Repository and Archive, University of Zurich ZORA URL: https://doi.org/10.5167/uzh-65998

Journal Article

Accepted Version

Originally published at:

Bartling, Björn (2012). Multi-tasking and inequity aversion in the linear-exponential-normal moral hazard model. Economics Letters, 116(3):523-525.

DOI: https://doi.org/10.1016/j.econlet.2012.04.010 


\title{
Multi-Tasking and Inequity Aversion in the \\ Linear-Exponential-Normal Moral Hazard Model
}

\author{
Björn Bartling*
}

April 2012

forthcoming in: Economics Letters

\begin{abstract}
This paper analyzes the impact of wage comparisons among inequity averse agents on optimal incentive intensities in a linear-exponential-normal moral hazard model with multi-tasking. We consider individual and team production tasks that differ in that only individual production causes wage inequality. If the tasks are substitutes in the agents' effort cost functions, the principal might want to balance incentives and reduce the agents' overall inequality exposure. We show that team production incentives can then be muted below the level that results from noisy measurement and risk aversion alone - even though team production does not cause wage inequality.
\end{abstract}

JEL Classification: D2, D8, M5

Keywords: multi-tasking, inequity aversion, wage comparison, moral hazard, team production

*University of Zurich, Department of Economics, Blümlisalpstrasse 10, 8006 Zurich, Switzerland, Tel.: +41-44-634-3722, Fax: +41-44-634-4907, e-mail: bjoern.bartling@econ.uzh.ch. 


\section{Introduction}

Much progress has been made in understanding optimal incentives in moral hazard models when agents have social preferences. ${ }^{1}$ The existing models consider environments where agents only conduct a single task. Often, however, principals must provide incentives for multiple tasks simultaneously. It is thus important to understand how social preferences affect optimal contracting in multi-tasking environments.

We analyze the impact of inequity aversion among agents (Fehr and Schmidt 1999) in a moral hazard model with multiple agents and multi-tasking (Holmström and Milgrom 1991). The existing multi-tasking literature analyzes the impact of differential measurement precision on optimal incentives. We focus on tasks, such as individual and team production, that differ in the extent of wage inequality that results from incentivizing the tasks. While the measurement precision of individual and team production should not differ systematically, a systematic difference with respect to wage inequality results when wages depend on measured output. A team output measure applies to all agents, but an individually produced output measure only applies to the respective agent. Hence, if the agents receive identical incentive contracts, wage inequality can result in individual production, but not in team production.

The highlighted difference between the two classes of tasks is, as such, irrelevant with purely self-interested agents. We show with inequity averse agents, in contrast, that differences in expected wage inequality can have effects on optimal incentive contracts comparable to those of differential measurement precision. Our approach in this paper is to document these effects in a model frame that places priority on yielding simple and clear closed form solutions, rather than in one that maximizes generality.

\section{Model}

Consider a principal and two identical agents $i, i \in\{1,2\}$, who devote effort $a_{i}$ to individual production tasks, measured by output $q_{i}=a_{i}+\varepsilon_{i}$, and to a team production task, measured by output $q_{t}=b_{i}+b_{j}+\varepsilon_{t}$, where $b_{i}$ and $b_{j}$ denote agent $i$ 's and $j$ 's effort devoted to team production, respectively. Error terms $\varepsilon_{i}$ and $\varepsilon_{t}$ are independent and

\footnotetext{
${ }^{1}$ See, e.g., Itoh (2004), Dur and Glazer (2008), and Neilson and Stowe (2010).
} 
drawn from normal distributions with mean zero. Importantly, with team production the same error term applies to both agents, because only joint output is measured. Chance variations thus cannot cause any inequality in measured team output. Since we are not interested in differences in measurement precision, we set the variance of both the individual and the team error term to $\sigma^{2}>0$.

The risk neutral principal maximizes expected profit, which is the sum of individual outputs and team output less wage payments. The agents have a constant absolute risk aversion utility function, $-\exp \left\{-\eta\left(w_{i}-\psi\left(a_{i}, b_{i}\right)-L_{i}\right)\right\}$, where $\eta>0$ is the risk aversion coefficient, $w_{i}$ agent $i$ 's wage, $\psi\left(a_{i}, b_{i}\right)$ the quadratic effort cost function, and $L_{i}$ agent $i$ 's expected loss from wage inequality, which is explained below.

Effort is non-contractible but agents can be remunerated as a function of individual and team output. We analyze symmetric, linear contracts, $w_{i}=r+v q_{i}+u q_{j}+v_{t} q_{t}$, with $i, j=1,2$ and $i \neq j$, where $r$ denotes a fixed payment, $v$ and $v_{t}$ are, respectively, the individual and team compensation coefficients, and $u$ is the coefficient of the respective other agent's individual output.

We assume that agents incur utility losses if their wages differ from each other and that the size but not the sign of the difference matters. ${ }^{2}$ In particular, we assume that agent $i$ cares about the expected loss from wage inequality, which, given $u \leq v,{ }^{3}$ is

$$
L_{i}=\alpha(v-u)\left(\int_{a_{i}-a_{j}}^{\infty}\left(z-a_{i}+a_{j}\right) f(z) d z+\int_{-\infty}^{a_{i}-a_{j}}\left(a_{i}-a_{j}-z\right) f(z) d z\right),
$$

where $z \equiv \varepsilon_{j}-\varepsilon_{i}$ and $f(z)$ is the p.d.f. of $z$, which is $z \sim N\left(0, \sigma_{z}^{2}\right)$ with $\sigma_{z}^{2}=2 \sigma^{2}$. The agents' sensitivity to inequality is denoted by $\alpha \geq 0$. The expected loss is taken ex-ante and independently from the expectation over wage levels, which implies risk neutrality with respect to wage inequality but risk aversion with respect to wage levels.

The principal maximizes expected gross profit minus expected wage payments by choice of $r, v, v_{t}$, and $u$. He must ensure the agents' participation and take into account

\footnotetext{
${ }^{2}$ In the notation of Fehr and Schmidt (1999), we assume $\alpha=\beta$. See Bartling (2011) for a singletasking version of the same model frame that allows for $\alpha \geq \beta$ and also for effort cost comparisons.

${ }^{3}$ It cannot be optimal to set $u>v$. To minimize risk exposure, $u$ must be set to zero, while $u=v$ minimizes inequality exposure. Further increasing $u$ thus cannot be optimal.
} 
that, given the contracts, the agents maximize their utility functions by choice of effort. By standard results, the principal's program can be written as

$$
\max _{v, v_{t}, u} a_{i}^{*}+b_{i}^{*}-\psi\left(a_{i}^{*}, b_{i}^{*}\right)-0.5 \eta\left(v^{2}+v_{t}^{2}+u^{2}\right) \sigma^{2}-L_{i}\left(a_{i}^{*}, a_{j}^{*}, v, u\right)
$$

subject to the incentive constraints for individual and team production

$$
v=\psi_{a_{i}^{*}} \quad \text { and } \quad v_{t}=\psi_{b_{i}^{*}}
$$

respectively. Subscripts to the cost function indicate partial derivatives. Coefficient $r$ must be finally set to ensure the agents' participation. We derive the symmetric equilibrium $a_{i}^{*}=a_{j}^{*}$. At $a_{i}=a_{j}$, as a consequence of symmetric inequity aversion, agents cannot affect expected wage inequality neither by marginally working harder nor by slacking off, i.e., $\partial L_{i} /\left.\partial a_{i}\right|_{a_{i}=a_{j}}=0$. Hence, as with purely self-interested agents, incentive constraints are given by (3). To ensure the agents' participation, however, agents must be compensated not only for effort costs and risk exposure but also for their expected loss from wage inequality in equilibrium. With symmetric effort choices, the equilibrium loss depends only on the relative size of the individual error terms and (1) can be written as

$$
L_{i}\left(v, u \mid a_{i}^{*}=a_{j}^{*}\right)=2 \alpha(v-u) \sigma / \sqrt{\pi}
$$

For the derivation see Appendix A1. The loss increases in the degree $\alpha$ of inequity aversion and in the measurement error $\sigma$, because with higher variance more probability mass is on larger wage differences. The smaller the difference $v-u$ of the individual compensation coefficients, the smaller the expected loss; it is zero if $u=v$.

\section{Contracts}

We first determine the coefficient $u$ linking an agent's wage to the respective other agent's individual output. Maximization of (2) with respect to $u$ yields

$$
u^{*}=\min \left[\frac{2 \alpha}{\eta \sigma \sqrt{\pi}}, v^{*}\right] \text {. }
$$


For the derivation see Appendix A2. To minimize risk, an agent's wage should be independent of the respective other agent's individual outcome, i.e., $u^{*}=0$. To minimize expected inequality, however, $u^{*}=v^{*}$ is optimal. Equation (5) shows the solution to the risk-inequality trade-off.

We can now derive the optimal incentive intensity. With multi-tasking, it is crucial how the agents' tasks interact in the effort cost functions. To highlight the point of this paper-differences in expected wage inequality have effects on optimal incentives similar to differences in measurement precision - we consider the case of perfect substitutes, i.e., $\psi(a, b)=\psi(a+b)$. Only the sum of $a+b$ can then be determined, and all cross-partial derivatives of the agents' cost function are identical. For notational convenience, we define $a+b=c$. If the principal wants the agents to engage in both tasks, incentive intensities cannot reflect differences in measurement precision nor differences in inequality exposure. The principal faces the single incentive constraint $v=v_{t}=\psi_{c}$. Maximization of (2) with respect to the incentive intensity then yields

$$
v^{*}=v_{t}^{*}=\max \left[\frac{1-2 \alpha \sigma \psi_{c c} / \sqrt{\pi}}{1+2 \eta \sigma^{2} \psi_{c c}}, \frac{1}{1+3 \eta \sigma^{2} \psi_{c c}}\right] .
$$

For the derivation see Appendix A3. The left entry in the brackets shows the optimal solution in case $u^{*}<v^{*}$, the right entry in case $u^{*}=v^{*}$. We summarize this finding in the following.

Proposition. With inequity averse agents, if the principal wants the agents to engage in both individual and team production, the incentive intensity for the team task can be muted below the level that results from noisy measurement and risk aversion alone in order to reduce expected wage inequality, even though team production never generates wage inequality.

The proof follows directly from the comparison of $v_{t}^{*}$ in (6) with the solution of the case with purely self-interested agents and perfect substitutability of effort, that is given by the left entry in (6) with $\alpha=0$. 


\section{Conclusions}

Our paper complements Holmström and Milgrom's (1991) well known finding that a muted incentive intensity for an easily measured task can be optimal, because an important competing task is difficult to measure. Homström and Milgrom explain the "puzzle [...] that employment contracts so often specify fixed wages and generally that incentives in firms appear to be so muted" (p. 24). Muted incentives appear puzzling, because often some tasks are easy to measure and thus appear well suited for high incentivization. We show that the multi-tasking framework can, in addition, explain muted incentives for an easily measured task not because a competing task is difficult to measure, but because a competing task generates wage inequality.

\section{Appendix}

\section{A.1 Derivation of the Expected Loss from Wage Inequality in Equation (4)}

Equation (4) is derived by integration of the expression in (1), given $a_{i}=a_{j}$.

$$
\begin{aligned}
L_{i} & =\alpha(v-u)\left(\int_{0}^{\infty} z f(z) d z+\int_{-\infty}^{0}-z f(z) d z\right) \\
& =2 \alpha(v-u) \int_{0}^{\infty} z f(z) d z=2 \alpha(v-u) \frac{1}{\sqrt{2 \pi} \sigma_{z}} \int_{0}^{\infty} z e^{-\frac{z^{2}}{2 \sigma_{z}^{2}}} d z \\
& =\lim _{y \rightarrow \infty} 2 \alpha(v-u) \frac{1}{\sqrt{2 \pi} \sigma_{z}}\left[-\sigma_{z}^{2} e^{-\frac{z^{2}}{2 \sigma_{z}^{2}}}\right]_{0}^{y}=2 \alpha(v-u) \frac{\sigma_{z}}{\sqrt{2 \pi}}=2 \alpha(v-u) \frac{\sigma}{\sqrt{\pi}}
\end{aligned}
$$

\section{A.2 Derivation of $u^{*}$ in Equation (5)}

The principal maximizes (2) with respect to $u$, where $L_{i}\left(a_{i}, a_{j}, v, u\right)$ is given by (4). The first-order condition is then given by

$$
\left(1-\psi_{a_{i}^{*}}\right) \frac{\partial a_{i}^{*}}{\partial u}-\eta u \sigma^{2}+\frac{2 \alpha \sigma}{\sqrt{\pi}}=0
$$

Since $\partial a_{i}^{*} / \partial u=0$, rearranging yields the left entry in (5). 


\section{A.3 Derivation of $v^{*}$ in Equation (6)}

With $v=v_{t}=\psi_{c}$, maximization of (2) with respect to $v$ requires in case $u^{*}<v$

$$
\left(1-\psi_{c_{i}^{*}}\right) \frac{\partial c_{i}^{*}}{\partial v}-2 \eta \sigma^{2} v-2 \alpha \sigma / \sqrt{\pi}=0
$$

and in case $u^{*}=v$

$$
\left(1-\psi_{c_{i}^{*}}\right) \frac{\partial c_{i}^{*}}{\partial v}-3 \eta \sigma^{2} v=0
$$

Substituting the first-order condition $v=\psi_{c_{i}^{*}}$ and using $\partial c_{i}^{*} / \partial v=1 / \psi_{c c}$, we get (6).

\section{References}

Bartling, B. (2011): "Relative or Team Performance Evaluation? Optimal Contracts for Other-Regarding Agents," Journal of Economic Behavior and Organization, 79(3), 183-93.

Dur, R., And A. Glazer (2008): "Optimal Contracts When a Worker Envies His Boss," Journal of Law, Economics, and Organization, 24(1), 120-37.

Fehr, E., And K. M. Schmidt (1999): "A Theory of Fairness, Competition, and Cooperation," Quarterly Journal of Economics, 114, 817-68.

Holmström, B., And P. Milgrom (1991): "Multitask Principal-Agent Analyses: Incentive Contracts, Asset Ownership, and Job Design," Journal of Law, Economics, and Organization, 7, 24-52.

Iтон, H. (2004): "Moral Hazard and Other-Regarding Preferences," Japanese Economic Review, 55, 18-45.

Neilson, W. S., And J. Stowe (2010): "Piece-Rate Contracts for Other-Regarding Workers," Economic Inquiry, 48(3), 575-86. 